\title{
Topside signature of medium-scale traveling ionospheric disturbances
}

\author{
E. S. Miller ${ }^{1}$, H. Kil ${ }^{1}$, J. J. Makela ${ }^{2}$, R. A. Heelis ${ }^{3}$, E. R. Talaat ${ }^{1,4}$, and A. Gross ${ }^{5}$ \\ ${ }^{1}$ Johns Hopkins University Applied Physics Laboratory, 11100 Johns Hopkins Road, Laurel, Maryland, USA \\ ${ }^{2}$ Department of Electrical and Computer Engineering, University of Illinois at Urbana-Champaign, Urbana, Illinois, USA \\ ${ }^{3}$ W. B. Hansen Center for Space Sciences, University of Texas at Dallas, Richardson, Texas, USA \\ ${ }^{4}$ NASA Headquarters, Washington, D.C., USA \\ ${ }^{5}$ Sunspots Bonaire, Bonaire \\ Correspondence to: E. S. Miller (ethan.miller@jhuapl.edu)
}

Received: 6 August 2013 - Revised: 23 June 2014 - Accepted: 3 July 2014 - Published: 18 August 2014

\begin{abstract}
Plasma blobs, localized plasma density enhancements that occur singularly or in periodic groups, have been observed by in situ sensors in the lower- and middle-latitude nighttime ionosphere. Traditionally, creation of blobs has been thought to be connected to equatorial plasma bubbles, which are localized plasma depletions. Here, we report the association of blobs with medium-scale traveling ionospheric disturbances (MSTIDs). On 17 January 2010, an allsky imager on the Caribbean island of Bonaire (geographic: $12.190^{\circ} \mathrm{N}, 68.244^{\circ} \mathrm{W}$; geomagnetic $22.46^{\circ} \mathrm{N}, 7.099^{\circ} \mathrm{E}$ ) observed a nighttime electrified MSTID propagating to the southwest. At the time of the MSTID's transit, the Coupled Ion-Neutral Dynamics Investigation instrument onboard the Communication/Navigation Outage Forecasting System satellite detected a group of blobs along the same geomagnetic flux tubes. The electron density variations measured at the satellite altitude, indicating the blobs, are anticorrelated with the intensity variations of the $630.0 \mathrm{~nm}$ dissociative recombination emission measured on the same magnetic field lines. This relationship is explained by a modulation of the $\mathrm{O}^{+}$profile altitude due to electric fields generated within the MSTID. This idea is supported by in situ measurements of the vertical ion velocity. We argue that common climatology between blobs and MSTIDs reported in the literature, as well as this coincident observation, suggest that blobs may be the in situ signature of MSTIDs in the topside ionosphere.
\end{abstract}

Keywords. Ionosphere (ionosphere-atmosphere interactions; ionospheric irregularities; plasma waves and instabilities)

\section{Introduction}

A number of reports of "plasma blobs", localized plasma density enhancements, observed by in situ plasma density probes have appeared in the literature (e.g., Oya et al., 1986; Le et al., 2003; Park et al., 2003, 2008; Klenzing et al., 2011b). Only a few studies have investigated other data sets in conjunction with the in situ plasma diagnostics. For example, Yokoyama et al. (2007) presented coincident observations of blobs and coherent very high frequency radar backscatter. Pimenta et al. (2007) presented plasma blobs associated with equatorial plasma depletions during a geomagnetic storm using ground-based airglow imaging and in situ plasma observations from the Defense Meteorological Satellite Program (DMSP). Likewise, Krall et al. (2010) demonstrated through modeling that the blob phenomenon could be attributed to equatorial plasma bubble activity. However, the specific plasma blob phenomenon investigated in the present report is not considered to be directly associated with equatorial plasma depletion activity (Kil et al., 2011; Choi et al., 2012).

Blobs occur in packets or as solitons with regular spacing on the order of hundreds of kilometers and exhibit increased density of as much as 3-4 times the background density (e.g., Oya et al., 1986). This periodicity and their proclivity for occurring at lower middle latitudes, near midnight in local time, and during solstices (e.g., Watanabe and Oya, 1986; Park et al., 2010) suggest that they might be associated with nighttime medium-scale traveling ionospheric 
disturbances (MSTIDs), which share a similar climatological distribution (Martinis et al., 2010; Makela and Miller, 2010; Duly et al., 2013). Nighttime MSTIDs are typically observed as one or more bands of decreased (or increased) $630.0 \mathrm{~nm}$ airglow intensity (e.g., Mendillo et al., 1997). They generally move westward and equatorward at phase velocities of $50-150 \mathrm{~m} \mathrm{~s}^{-1}$ (e.g., Garcia et al., 2000). All of these features suggest that blobs and MSTIDs at least share some conditions conducive to their formation and perhaps might be different observational manifestations of the same physical process.

Saito et al. (1995) identified electric field variations at conjugate middle-latitude locations in DE-2 data that Otsuka et al. (2004) later attributed to MSTIDs when demonstrating the conjugacy of MSTIDs in airglow images. Shiokawa et al. (2003) demonstrated that an MSTID observed in the airglow produced an electric field and density perturbations at the $847 \mathrm{~km}$ altitude of the DMSP F15 spacecraft as it crossed the same geomagnetic field lines. These observations were also consistent with electric field measurements made with the Arecibo incoherent scatter radar by Kelley et al. (2000).

The fact that MSTIDs appear in conjugate hemispheres suggests that they are electrodynamic features that evolve on temporal and spatial scales that are sufficiently large to consider the geomagnetic field lines to be equipotential. Kelley (2011) asserts that this kind of nighttime MSTID is due to a gravity wave neutral wind field that experiences minimum Joule damping by the cancellation of the dynamo $\boldsymbol{U} \times \boldsymbol{B}$ current with a polarization electric field and associated $\boldsymbol{E} \times \boldsymbol{B}$ drift. Furthermore, the combination of equipotential geomagnetic field lines and internal $\boldsymbol{E}$ fields means that the $\boldsymbol{E} \times \boldsymbol{B}$ drift (and subsequent perturbation in ion density) occurs all along the geomagnetic field line even when the interaction with the neutral wind field is only occurring in one hemisphere and possibly at lower altitude.

In this paper, we leverage the conjugacy argument outlined above to present evidence that an MSTID observed with an airglow imager on the ground produces the blob or localized enhancement signature in the in situ plasma density observations along the same geomagnetic field line. Furthermore, we identify characteristics of the density enhancement that are consistent with theories of MSTID behavior. We will begin with a description of the instrumentation employed, followed by a brief outline of the data processing, a discussion of the data and their implications, and finally a conclusion.

\section{Instrumentation}

A wide-field Portable Ionospheric Camera and Small-Scale Observatory (PICASSO) was installed on the Caribbean island of Bonaire (geographic: $12.190^{\circ} \mathrm{N}, 68.244^{\circ} \mathrm{W}$; geomagnetic $22.46^{\circ} \mathrm{N}, 7.099^{\circ} \mathrm{E}$ ) in March 2009 for studies of middle-latitude plasma structure in the southern Caribbean. This PICASSO system collected $90 \mathrm{~s}$ exposures of the
$630.0 \mathrm{~nm} \mathrm{O}\left({ }^{1} \mathrm{D}\right)$ dissociative recombination emission line, as well as the $777.4 \mathrm{~nm}$ radiative recombination line and another wavelength $(643.4 \mathrm{~nm})$ for sampling the background for photometric correction of the $630.0 \mathrm{~nm}$ images.

The Coupled Ion-Neutral Dynamics Investigation (CINDI) package aboard the Communication/Navigation Outage Forecasting System (C/NOFS) spacecraft consists of the Neutral Wind Meter (NWM) and Ion Velocity Meter (IVM). C/NOFS is in a low-inclination elliptical orbit $\left(13^{\circ}\right)$ orbit whose altitude varies in the range between 400 and $850 \mathrm{~km}$. The data sampling cadence of the CINDI retarding potential analyzer (RPA) in the IVM is $0.5 \mathrm{~s}$.

\section{Data}

On 17 January 2010, a sequence of relatively cloud-free (determined by visual inspection) images of the $630.0 \mathrm{~nm}$ airglow line indicated nighttime MSTID bands oriented from northwest (NW) to southeast (SE) and propagating to the southwest (SW) over Bonaire beginning around 00:38 UT (20:38 LT, 16 January 2010). In this paper, we use the term "MSTID" to collectively refer to a series of alternating light and dark bands observed to propagate coherently in the airglow with the geographic and propagation characteristics outlined previously. This time period was characterized by very quiet geomagnetic conditions $(\mathrm{Kp} \leq 1-$ during all of 16 and 17 January 2010), which solidifies the argument that the observed structure is an MSTID and not the somewhat unusual storm-time observation of an equatorial plasma depletion (bubble) in retrograde (westward) drift. Cloud cover between the commencement of data collection at 00:00 UT and 01:00 UT partially obscured the appearance and progression of the MSTID structure as it drifted into view from the northwest around 00:38 UT. Because the $630.0 \mathrm{~nm}$ airglow emanates from a relatively thin $(50 \mathrm{~km}$ or so) layer around $250 \mathrm{~km}$ altitude, images collected on the ground can be spatially warped and projected on a shell to create a twodimensional map of ionospheric structure. Figure 1 presents a series of these airglow maps showing the progression of the MSTID from NE to SW between 01:06 and 01:46 UT.

At 01:35 UT, C/NOFS passed just to the south of Bonaire at an altitude of $470 \mathrm{~km}$, detecting a series of plasma blobs with the CINDI instrument. Farley $(1959,1960)$ showed that electric fields of sufficient spatial scale (larger than $10 \mathrm{~km}$ or so) map efficiently along the geomagnetic field in the F region. We can leverage this fact to fold the in situ and airglow observations into the same two-dimensional coordinate system for direct comparison, as done in previous cases (e.g., Miller and Makela, 2008). The location of each in situ sample is mapped down to the airglow layer (nominally at $250 \mathrm{~km}$ altitude) along the geomagnetic field using the International Geomagnetic Reference Field (IGRF-11) (Finlay et al., 2010). 

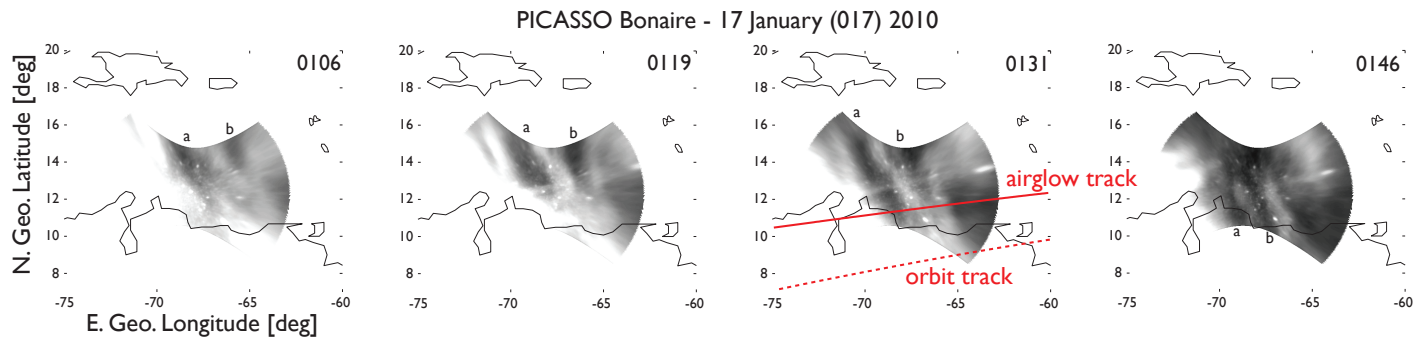

Figure 1. Sequence of airglow images collected by the all-sky airglow imager in Bonaire. Images have been spatially calibrated to the star field and mapped to the nominal airglow layer at $250 \mathrm{~km}$ altitude. Dark MSTID bands with wavefronts aligned NW to SE and propagating from NE to SW are identified as "a" and "b". At 01:31 UT, the C/NOFS orbit path at $470 \mathrm{~km}$ (dotted) is shown along with the geomagnetically mapped path (solid) in the airglow layer at $250 \mathrm{~km}$ altitude.

Figure 2 shows the C/NOFS orbit segment over the Bonaire imager in two dimensions: (panel a) $\mathrm{O}^{+}$(solid) and $\mathrm{H}^{+}$(dotted) densities at 01:35 UT on 17 January 2010, as well as $\mathrm{O}^{+}$(dashed) density from the previous orbit pass at 23:51 UT on 16 January 2010; (panel b) ion drift velocities; and (panel c) the along-track interpolated airglow; all are as a function of the C/NOFS geographic longitude. The ion velocities are expressed in geomagnetic coordinates. $V_{\text {merid }}$ and $V_{\text {zonal }}$ are the components perpendicular to the magnetic field line in the magnetic meridional (positive is upward) and zonal (positive is eastward) directions, respectively. The dashed curve in Fig. 2 a shows the $\mathrm{O}^{+}$density around 23:51 UT on the previous C/NOFS orbit. The observations of the dashed curve were made a few degrees south from the observations of the solid curve. Only very slight structure appears in the $\mathrm{O}^{+}$density during the earlier orbit. The shaded regions approximately define the locations of the two clearest blobs identified from the CINDI ion density data full width at half maximum (FWHM). We note the fact that MSTIDs appeared beginning at 00:38 UT over Bonaire, after the earlier C/NOFS pass. Although there exists about $90 \mathrm{~min}$ of uncertainty on the appearance time of blobs (the observation time difference between the dashed and solid curves), detection of blobs at the time of the MSTID transit of connected field lines supports the hypothesis that MSTIDs can produce the blob density perturbation signature.

A second event was observed on 21 January 2010 at 02:45 UT. In the same manner as Fig. 2, this event is depicted in Fig. 3. This event is a little different from the first in that it exhibits up to 1 degree of offset between the airglow maximum and the corresponding $\mathrm{CINDI}\left[\mathrm{O}^{+}\right]$minimum. The airglow image used in this example is also the first collected from the night, about $40 \mathrm{~min}$ after $\mathrm{C} / \mathrm{NOFS}$ had passed. This offset is discussed further in the following section.

\section{Discussion}

The $630.0 \mathrm{~nm}$ airglow emission line results from the dissociative recombination of $\mathrm{O}_{2}^{+}$produced by charge exchange between $\mathrm{O}^{+}$and $\mathrm{O}_{2}$ :
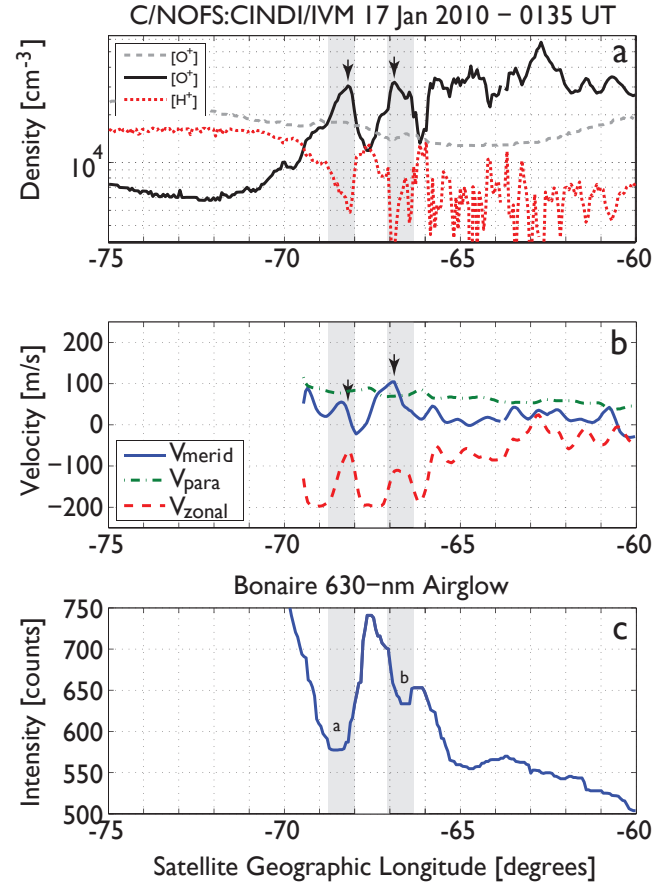

Figure 2. Three-panel plot comparing in situ plasma observations with airglow observations as a function of C/NOFS geographic longitude: (a) $\mathrm{O}^{+}$(solid) and $\mathrm{H}^{+}$(dotted) densities as well as the $\mathrm{O}^{+}$ density from the prior orbit (dashed); (b) ion drift velocities; and (c) the along-track interpolated airglow. Densities are in $\mathrm{cm}^{-3}$ and velocities are in $\mathrm{ms}^{-1}$, with $V_{\text {merid }}$ positive upward, $V_{\text {para }}$ positive northward, and $V_{\text {zonal }}$ positive eastward. Image intensities are in CCD counts and are not photometrically calibrated for this analysis. The velocity data are not plotted for periods when the C/NOFS spacecraft was above the $\mathrm{O}^{+} / \mathrm{H}^{+}$transition altitude.

$\mathrm{O}^{+}+\mathrm{O}_{2} \rightarrow \mathrm{O}_{2}^{+}+\mathrm{O}$

$\mathrm{O}_{2}^{+}+e^{-} \rightarrow \mathrm{O}\left({ }^{1} D\right)+\mathrm{O}\left({ }^{3} P\right)$

The generation of $\mathrm{O}_{2}^{+}$(and, hence, the $630.0 \mathrm{~nm}$ emission from $\left.\mathrm{O}\left({ }^{1} \mathrm{D}\right)\right)$ therefore has a joint dependence on both the $\mathrm{O}^{+}$ and $\mathrm{O}_{2}$ densities. For a constant background concentration of 
$\mathrm{O}_{2}$, this joint dependence yields an increase (brightening) in $630.0 \mathrm{~nm}$ emission when the $\mathrm{O}^{+}$is driven to lower altitude and a decrease (darkening) in emission when $\mathrm{O}^{+}$is driven upward.

The simultaneous increases in $\mathrm{O}^{+}$and decreases in $\mathrm{H}^{+}$observed by CINDI and illustrated in Figs. 2a and 3a are also consistent with electrodynamic uplift of the $\mathrm{F}$ region (Kil et al., 2011): as the F region is driven upward, CINDI detects the $\mathrm{O}^{+}$density increase and $\mathrm{H}^{+}$density decrease because the C/NOFS spacecraft is situated on the topside of the $\mathrm{O}^{+}$ profile and the bottomside of the $\mathrm{H}^{+}$profile. As the $\mathrm{O}^{+}$-rich $F$ region peak is driven upward, the $\mathrm{H}^{+}$population is driven above the spacecraft altitude. Likewise, a decrease in $\mathrm{O}^{+}$and an increase in $\mathrm{H}^{+}$corresponds to electrodynamic lowering. The essence of this work is these in situ uplifts (lowerings) on the topside are coincident with the airglow uplifts (lowerings) along the same geomagnetic flux tubes on the bottomside.

To substantiate our claim that the spacecraft was operating at the transition height, we note that Heelis et al. (2009) reported the transition altitude as low as $450 \mathrm{~km}$ at night in July/August 2008. Aponte et al. (2013) confirmed this again in October 2009 using the Arecibo incoherent scatter radar (ISR), which is not far from the observations presented in this paper. Arecibo was not active in incoherent scatter mode on 17 January 2010. However, it was active on 21 January 2010 and the transition altitude of around $525 \mathrm{~km}$ was deduced from CINDI versus around $550-575 \mathrm{~km}$ from the ISR. Finally, this is also consistent with globally averaged CINDI observations reported by Klenzing et al. (2011a) from 2009 and 2010, which showed the transition height between 500 and $600 \mathrm{~km}$ in the pre-midnight local time sector.

In the case of plasma bubbles detected in the topside, the $\mathrm{O}^{+}$and $\mathrm{H}^{+}$densities are positively correlated. This phenomenon can be explained by noting that a plasma bubble is a depleted wedge that extends from the bottomside to the topside, whereas a blob is an altitude perturbation in the density profile. The anticorrelation between the $\mathrm{O}^{+}$and $\mathrm{H}^{+}$densities at the locations of blobs indicates that a moderate modulation of the $\mathrm{F}$ region height is the cause of these blobs (Oya et al., 1986; Le et al., 2003). As Fig. 2a shows, ion density irregularities are essentially absent at the same longitude on the earlier orbit. This, in addition to the coincident airglow images showing the structures drifting to the southwest, suggests that equatorial plasma bubbles (which would drift to the east) are not the source of these blobs.

In Fig. $2 b$, the vertical ion velocity change is shown to be well correlated with the change of the $\mathrm{O}^{+}$density; blobs appear at the locations where the upward ion velocity is enhanced. The zonal ion velocity also shows only fluctuations at the locations where blobs are detected. However, the parallel velocity component shows a minor variation. The fluctuations of the perpendicular components of the velocity at the locations of blobs indicate that creation of blobs is associated with convective electric fields generated in the MSTID at lower altitudes.
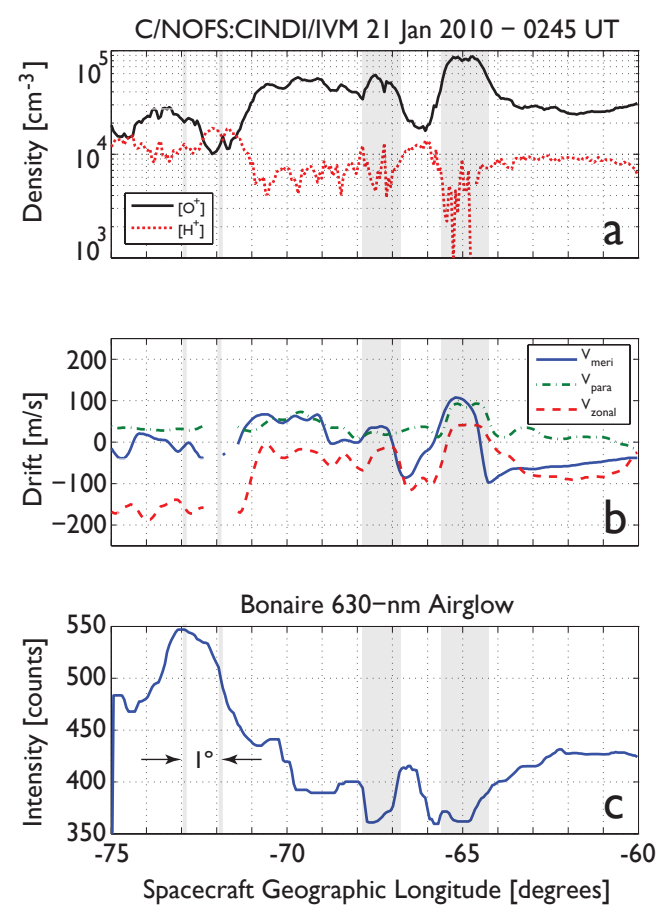

Figure 3. Another nearly coincident observation by C/NOFS and PICASSO/Bonaire on 21 January 2009. Plot format is the same as Fig. 2. In this case, the brightness peaks in the airglow are offset up to 1 degree in longitude from the decreases (increases) in the $\mathrm{O}^{+}\left(\mathrm{H}^{+}\right)$density. The $\mathrm{C} / \mathrm{NOFS}$ pass occurred about $40 \mathrm{~min}$ before airglow imaging commenced on this night. Again, the CINDI/IVM velocity data are not plotted for periods when the C/NOFS spacecraft was above the $\mathrm{O}^{+} / \mathrm{H}^{+}$transition altitude.

Figure 4 schematically illustrates the effect of the $\mathrm{F}$ region height change along a geomagnetic field line on the $\mathrm{O}^{+}$and $\mathrm{H}^{+}$density and airglow intensity. Uplift of the ionosphere causes the $\mathrm{O}^{+}$increase and the $\mathrm{H}^{+}$to decrease at the altitude of $\mathrm{C} / \mathrm{NOFS}$, whereas it causes the $\mathrm{O}^{+}$decrease at the altitude where the $630.0 \mathrm{~nm}$ emission is produced. As a result, C/NOFS observes the $\mathrm{O}^{+}$increase and $\mathrm{H}^{+}$decrease (a blob) and the imager observes the $630.0 \mathrm{~nm}$ emission depletion by the reduction of the charge exchange rate between $\mathrm{O}^{+}$and $\mathrm{O}_{2}$ (and subsequent reduction in $\mathrm{O}\left({ }^{1} \mathrm{D}\right)$ production and $630.0 \mathrm{~nm}$ emission). Thus the anticorrelation between the topside $\mathrm{O}^{+}$ density and bottomside $630.0 \mathrm{~nm}$ emission is explained by modulation of the F region height. This explains the out-ofphase relationship between airglow intensity and $\mathrm{O}^{+}$density in Fig. 2a and c.

The second example, in Fig. 3, exhibits an offset between the C/NOFS observations of density and the PICASSO/Bonaire airglow enhancements, which were collected about 40 min after the spacecraft passed the site. An MSTID crest (trough) with a phase speed of $100 \mathrm{~m} \mathrm{~s}^{-1}$ to the southwest will cover 2.3 degrees of longitude in $1 \mathrm{~h}$. In this case, covering about 1.0 degrees in 40 min suggests a phase 

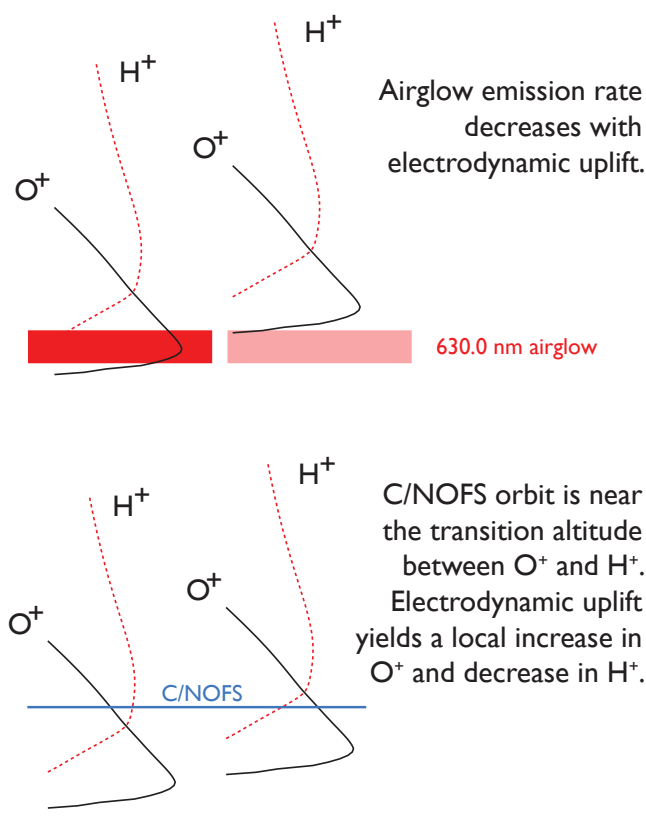

Figure 4. Schematic illustration of the connection between the change in $\mathrm{O}^{+}$and $\mathrm{H}^{+}$density in the bottomside and topside ionosphere as driven by an electric field that maps along the geomagnetic field. For upward $\boldsymbol{E} \times \boldsymbol{B}$ drift, the bottomside $\mathrm{O}^{+}$profile is driven to higher altitude reducing charge exchange with $\mathrm{O}_{2}$ thereby reducing the $\mathrm{O}\left({ }^{1} \mathrm{D}\right)$ population available to produce $630.0 \mathrm{~nm}$. Likewise, at the C/NOFS altitude near the $\mathrm{O}^{+} / \mathrm{H}^{+}$transition height, upward $\boldsymbol{E} \times \boldsymbol{B}$ drifts produce an increase in $\mathrm{O}^{+}$and a decrease $\mathrm{H}^{+}$. The opposite occurs for a downward $\boldsymbol{E} \times \boldsymbol{B}$ drift: the $\mathrm{O}^{+}$profile is driven into a region of more $\mathrm{O}_{2}$ and the bottomside $630.0 \mathrm{~nm}$ emission increases and at the spacecraft altitude, the $\mathrm{H}^{+}$density increases and the $\mathrm{O}^{+}$density decreases.

speed of about $65 \mathrm{~m} \mathrm{~s}^{-1}$, which is consistent with MSTID behavior.

We note here that the phase speed of an MSTID refers to the propagation of the entire structure of alternating bands, a parameter which is derived from a time offset in density (or airglow) observations. However, detrending $v_{\text {meri }} \approx$ $-25 \mathrm{~m} \mathrm{~s}^{-1}$ and $v_{\text {zonal }} \approx-50 \mathrm{~m} \mathrm{~s}^{-1}$ from the in situ drifts of Fig. $3 b$ results in a little less than $60 \mathrm{~m} \mathrm{~s}^{-1}$, which is quite consistent with the phase velocity estimate. Likewise, from Fig. 1 we see that the MSTID structure propagates about 4 degrees in longitude in $40 \mathrm{~min}$, corresponding to a westward (zonal) phase velocity component of $180 \mathrm{~m} \mathrm{~s}^{-1}$, which is consistent with Fig. 2b, where the detrended in situ $v_{\text {zonal }} \approx-150 \mathrm{~m} \mathrm{~s}^{-1}$.

Although Saito et al. (1995) do not report density fluctuations accompanying electric field perturbations, they refer to observations of density perturbations coincident with electric field variations observed at the OGO 6 spacecraft in earlier work by Holtet et al. (1977). All events examined by Holtet et al. (1977) appear to be associated with equatorial plasma depletions and related phenomena, even when they extend to $35^{\circ}$ invariant latitude. However, Shiokawa et al. (2003) observed density variations in DMSP F15 SSIES observations on the topside ( $847 \mathrm{~km}$ altitude) associated with MSTIDs observed in the $630.0 \mathrm{~nm}$ airglow at $250 \mathrm{~km}$.

A further curious difference between the present examples and Klenzing et al. (2011b) is the considerably higher parallel (to the geomagnetic field) velocities reported in the latter. We suspect that there may be a large variability in these parallel drifts depending on solar-geomagnetic conditions and the precise observation geometry. A meridional $\boldsymbol{E} \times \boldsymbol{B}$ drift at off-equatorial latitudes can be balanced by field-aligned diffusion that drives a large parallel drift. This may explain why some MSTIDs produce large drifts but imperceptible density perturbations in spacecraft in situ observations.

Klenzing et al. (2011b) also noted an offset in longitude between the maximum vertical/meridional velocity perturbations and plasma enhancement maxima. This offset is also visible in Fig. 2a and b, where it is indicated by arrowheads on the left-most blob. It is not evident on the right-hand blob. A trivial transport model (not elaborated herein although physically intuitive) suggests that if the blobs are the signature of $\boldsymbol{E} \times \boldsymbol{B}$ drifts, the velocity perturbation leads the density perturbation. That is, if we take the time integral of a cosinusoidal drift to obtain density, the density is sinusoidal with the cosine (drift) leading the sine (density). Because electrified nighttime MSTIDs have southwestward phase velocity, we expect to observe peak velocities to the west of the fieldmapped MSTID crests (density enhancements). This is consistent with the observations in both Fig. 2a and b and with the work of Klenzing et al. (2011b).

\section{Conclusions}

In this paper, we have examined coincident observations from an airglow imager in the Caribbean and the CINDI in situ plasma density and velocity instrument on the C/NOFS spacecraft. MSTIDs were observed in the bottomside $630.0 \mathrm{~nm}$ airglow at the same time and geomagnetically mapped location as plasma density enhancements (plasma blobs) were observed in situ on the topside with CINDI. This suggests that local (off-equator) layer height changes due to a passing MSTID are related to density perturbations at the spacecraft. The drift and density perturbation characteristics of these blobs are consistent with a structure driven by the MSTID.

Acknowledgements. This work was supported by National Science Foundation (NSF) grant AGS-1237276. E. S. Miller acknowledges further support from NSF award AGS-0924914. J. J. Makela acknowledges support from NSF CAREER award ATM-0644654. R. A. Heelis is supported by NASA grant NNX10AT02G to the University of Texas at Dallas. E. R. Talaat acknowledges support from NSF award ATM-0838142. We thank Michael Copini for technical support for the PICASSO system in Bonaire and Telfonia Bonairi- 
ano for providing Internet access for this system.

Topical Editor R. Stoneback thanks two anonymous referees for their help in evaluating this paper.

\section{References}

Aponte, N., Brum, C. G. M., Sulzer, M. P., and González, S. A.: Measurements of the $\mathrm{O}^{+}$to $\mathrm{H}^{+}$transition height and ion temperatures in the lower topside ionosphere over Arecibo for equinox conditions during the 2008-2009 extreme solar minimum, J. Geophys. Res.-Space, 118, 4465-4470, doi:10.1002/jgra.50416, 2013.

Choi, H.-S., Kil, H., Kwak, Y.-S., Park, Y.-D., and Cho, K.S.: Comparison of the bubble and blob distributions during the solar minimum, J. Geophys. Res., 117, A04314, doi:10.1029/2011JA017292, 2012.

Duly, T. M., Chapagain, N. P., and Makela, J. J.: Climatology of nighttime medium-scale traveling ionospheric disturbances (MSTIDs) in the Central Pacific and South American sectors, Ann. Geophys., 31, 2229-2237, doi:10.5194/angeo-31-22292013, 2013.

Farley, D. T.: A theory of electrostatic fields in a horizontally stratified ionosphere subject to a vertical magnetic field, J. Geophys. Res., 64, 1225-1233, 1959.

Farley, D. T.: A theory of electrostatic fields in the ionosphere at non-polar geomagnetic latitudes, J. Geophys. Res., 65, 869-877, 1960.

Finlay, C. C., Maus, S., Beggan, C. D., Bondar, T. N., Chambodut, A., Chernova, T. A., Chulliat, A., Golovkov, V. P., Hamilton, B., Hamoudi, M., Holme, R., Hulot, G., Kuang, W., Langlais, B., Lesur, V., Lowes, F. J., Lühr, H., Macmillan, S., Mandea, M., McLean, S., Manoj, C., Menvielle, M., Michaelis, I., Olsen, N., Rauberg, J., Rother, M., Sabaka, T. J., Tangborn, A., TøffnerClausen, L., Thébault, E., Thomson, A. W. P., Wardinski, I., Wei, Z., and Zvereva, T. I.: International Geomagnetic Reference Field: the eleventh generation, Geophys. J. Int., 183, 1216-1230, 2010.

Garcia, F. J., Kelley, M. C., Makela, J. J., and Huang, C.-S.: Airglow observations of mesoscale low-velocity traveling ionospheric disturbances at midlatitudes, J. Geophys. Res., 105, 1840718415, 2000.

Heelis, R. A., Coley, W. R., Burrell, A. G., Hairston, M. R., Earle, G. D., Perdue, M. D., Power, R. A., Harmon, L. L., Holt, B. J., and Lippincott, C. R.: Behavior of the $\mathrm{O}^{+} / \mathrm{H}^{+}$transition height during the extreme solar minimum of 2008, Geophys. Res. Lett., 36, L00C03, doi:10.1029/2009GL038652, 2009.

Holtet, J. A., Maynard, N. C., Heppner, J. P., and Aponte, N.: Variational electric fields at low latitudes and their relation to spread$F$ and plasma irregularities, J. Atmos. Terr. Phys., 39, 247-262, 1977.

Kelley, M. C.: On the origin of mesoscale TIDs at midlatitudes, Ann. Geophys., 29, 361-366, doi:10.5194/angeo-29-361-2011, 2011.

Kelley, M. C., Makela, J. J., Saito, A., Aponte, N., Sulzer, M., and González, S.: On the electrical structure of airglow depletion/height layer bands over Arecibo, Geophys. Res. Lett., 27, 2837-2840, 2000.

Kil, H., Choi, H.-S., Heelis, R. A., Paxton, L. J., Coley, W. R., and Miller, E. S.: Onset conditions of bubbles and blobs: A case study on 2 March 2009, Geophys. Res. Lett., 38, L06101, doi:10.1029/2011GL046885, 2011.

Klenzing, J., Simões, F. S., Ivanov, S., Heelis, R. A., Bilitza, D., Pfaff, R., and Rowland, D.: Topside equatorial ionospheric density and composition during and after extreme solar minimum, J. Geophys. Res., 116, A12330, doi:10.1029/2011JA017213, 2011a.

Klenzing, J. H., Rowland, D. E., Pfaff, R. F., Le, G., Freudenreich, H., Haaser, R. A., Burrel, A. G., Stoneback, R. A., Coley, W. R., and Heelis, R. A.: Observations of low-latitude plasma density enhancements and their associated plasma drifts, J. Geophys. Res., 116, A09324, doi:10.1029/2011JA016711, $2011 \mathrm{~b}$.

Krall, J., Huba, J. D., Joyce, G., and Yokoyama, T.: Density enhancements associated with equatorial spread F, Ann. Geophys., 28, 327-337, doi:10.5194/angeo-28-327-2010, 2010.

Le, G., Huang, C.-S., Pfaff, R. F., Su, S.-Y., Yeh, H.-C., Heelis, R. A., Rich, F. J., and Hairston, M.: Plasma density enhancements associated with equatorial spread F: ROCSAT1 and DMSP observations, J. Geophys. Res., 108, 1318, doi:10.1029/2002JA009592, 2003.

Makela, J. J. and Miller, E. S.: Influences on the Development of Equatorial Plasma Bubbles: Insights from a Long-Term Optical Dataset, in: IAGA Division II Book, edited by: Bhattacharyya, A., 2010.

Martinis, C., Baumgardner, J., Wroten, J., and Mendillo, M.: Seasonal dependence of MSTIDs obtained from $630.0 \mathrm{~nm}$ airglow imaging at Arecibo, Geophys. Res. Lett., 37, L11103, doi:10.1029/2010GL043569, 2010.

Mendillo, M., Baumgardner, J., Nottingham, D., Aarons, J., Reinisch, B., Scali, J., and Kelley, M.: Investigations of thermospheric-ionospheric dynamics with 6300-A images from the Arecibo Observatory, J. Geophys. Res., 102, 7331-7343, 1997.

Miller, E. S. and Makela, J. J.: A multi-instrument technique for localization of scintillation-causing regions in the equatorial ionosphere, J. Geophys. Res., 113, A12303, doi:10.1029/2008JA013144, 2008.

Otsuka, Y., Shiokawa, K., Ogawa, T., and Wilkinson, P.: Geomagnetic conjugate observations of medium-scale traveling ionospheric disturbances at midlatitude using allsky airglow imagers, Geophys. Res. Lett., 31, L15803, doi:10.1029/2004GL020262, 2004.

Oya, H., Takahashi, T., and Watanabe, S.: Observation of Low Latitude Ionosphere by the Impedance Probe on Board the Hinotori Satellite, J. Geomag. Geoelectr., 38, 111-123, 1986.

Park, J., Min, K. W., Lee, J.-J., Kil, H., Kim, V. P., Kim, H.-J., Lee, E., and Lee, D. Y.: Plasma blob events observed by KOMPSAT-1 and DMSP F15 in the low latitude nighttime upper ionosphere, Geophys. Res. Lett., 30, 2114, doi:10.1029/2003GL018249, 2003.

Park, J., Stolle, C., Lühr, H., Rother, M., Su, S.-Y., Min, K. W., and Lee, J.-J.: Magnetic signatures and conjugate features of lowlatitude plasma blobs as observed by the CHAMP satellite, J. Geophys. Res., 133, A09313, doi:10.1029/2008JA013211, 2008.

Park, J., Lühr, H., Min, K. W., and Lee, J.-J.: Plasma density undulations in the nighttime mid-latitude F-region as observed by CHAMP, KOMPSAT-1, and DMSP F15, J. Atmos. Sol.-Terr. Phys., 72, 183-192, 2010. 
Pimenta, A. A., Sahai, Y., Bittencourt, J. A., and Rich, F. J.: Ionospheric plasma blobs observed by OI $630 \mathrm{~nm}$ all-sky imaging in the Brazilian tropical sector during the major geomagnetic storm of April 6-7, 2000, Geophys. Res. Lett., 34, L02820, doi:10.1029/2006GL028529, 2007.

Saito, A., Iyemori, T., Sugiura, M., Maynard, N. C., Aggson, T. L., Brace, L. H., Takeda, M., and Yamamoto, M.: Conjugate occurrence of the electric field fluctuations in the nighttime midlatitude ionosphere, J. Geophys. Res., 100, 21439-21451, 1995.

Shiokawa, K., Otsuka, Y., Ihara, C., and Ogawa, T.: Ground and satellite observations of nighttime medium-scale traveling ionospheric disturbance at midlatitude, J. Geophys. Res., 108, 1145, doi:10.1029/2002JA009639, 2003.
Watanabe, S. and Oya, H.: Occurrence Characteristics of Low Latitude Ionosphere Observed by the Impedance Probe on Board the Hinotori Satellite, J. Geomag. Geoelectr., 38, 125-149, 1986.

Yokoyama, T., Su, S.-Y., and Fukao, S.: Plasma blobs and irregularities concurrently observed by ROCSAT-1 and Equatorial Atmosphere Radar, J. Geophys. Res., 112, A05311, doi:10.1029/2006JA012044, 2007. 